\title{
Study of scalar gradient fields by geometric measure theory
}

\author{
Jörg Schumacher \\ Fachbereich Physik, Philipps-Universität, D-35032 Marburg, Germany
}

(November 3, 2018)

\begin{abstract}
Upper bounds of the Hausdorff volume of scalar gradient field graphs are derived by means of geometric measure theory. The approach reproduces that scalar gradient fields along a mean imposed scalar gradient become space filling for sufficiently high values of Schmidt numbers $S c$. The bounds are consistent with findings from recent high-resolution numerical experiments for $1 \leq S c \leq 64$, but too rough when compared with numerical simulations. A Reynolds number dependence of the bounds is found due to the additional scalar gradient stretching term in the equation of motion.

PACS: 47.27.Eq, 47.53.+n, 02.40.-k
\end{abstract}

Recent direct numerical simulations (DNS) suggested that the passive scalar mixing in a turbulent flow becomes more isotropic when the Schmidt number, $S c=\nu / \kappa$, is increased to values larger than unity, but the Taylor Reynolds number, $R_{\lambda}$, of the advecting turbulent flow is kept constant $[1,2]$. Here $\nu$ is the kinematic viscosity of the fluid and $\kappa$ the diffusivity of the passive scalar field $\theta(\mathbf{x}, t)$. The scalar was driven by a mean scalar gradient, $\mathbf{G}=G \mathbf{e}_{\alpha}$, in both simulations which causes deviations from isotropy of the small-scale statistics. For increasing $S c$, scalar filaments can be advected to ever finer scales which steepens up the local gradients, $g_{i}=\partial_{i} \theta$. Large gradients (or fronts) that are aligned with the mean, i.e. $g_{\alpha}$, occur already for $S c \sim 1$ or even below and are associated with characteristic scalar structures, so-called ramps and cliffs (for further references, see [3]). A return to isotropic mixing is then thought as a growing compensation of those pronounced positive fronts by an increasing number of steep negative gradients with increasing $S c$. Consequently, the probability density function of $g_{\alpha}$ gets more symmetric tails and odd order derivative moments decay.

Our understanding of this process for advection in Navier-Stokes flows is still incomplete and an investigation of geometric properties of scalar gradient fields was started therefore recently [4]. Fractal and multifractal properties of scalar gradient level sets and related quantities were studied there in a series of high-resolution DNS where, e.g., a higher degree of local isotropy was found to be related to a flater spectrum of generalized (multifractal) dimensions.

On the theoretical side, concepts of geometric measure theory [5] were used sucessfully for scalars in turbulence $[6,7]$ and relations to the scaling behavior of low-order structure functions were established. An extension of the framework discussed the dependence of the geometric properties of scalar level sets on Schmidt (or Prandtl) number and on spatial separation scales, respectively [8]. The purpose of the present brief report is to step in at this point and to extend the approach to scalar gradient fields. We will focus on the physics in the Batchelor regime of scalar turbulence where the advecting flow is in its viscous subrange [9]. Scales below the Kolmogorov dissipation scale of fluid turbulence, $\eta=\left(\nu^{3} / \epsilon\right)^{1 / 4}$, but larger than the Batchelor scale $\eta_{B}=\eta / S c^{1 / 2}$ are considered. $\epsilon$ is the energy dissipation rate of the flow. We will derive an upper bound for the scaling dimension of the Hausdorff volume of the scalar gradient field graph. This can give us an idea of how the gradients are distributed spatially and how this distribution depends on $S c$. Our findings will be compared finally with high resolution numerical data for Schmidt numbers between 1 and 64 to test the sensitivity of the derived scaling dimension bounds.

The geometric measure theory generalizes concepts of differential geometry to non-smooth hypersurfaces embedded in an Euclidian space. The central object is the graph of the field under consideration, which is defined as

$$
\Gamma_{r}=\left\{\left(\mathbf{x}, g_{\alpha}\right) \mid \mathbf{x} \in B_{r} \subset \mathrm{R}^{3}, g_{\alpha}=g_{\alpha}(\mathbf{x})\right\} .
$$

In case of a smooth field this is a three-dimensional hypersurface embedded in four-dimensional space. In Fig. 1 such graph is shown over a two-dimensional plane that is vertical to the direction of $\mathbf{G}$. We observe that the hypersurface is strongly folded and rough for larger scales thus suggesting fractal properties.

The Hausdorff dimension of such a graph, $D_{H}$, (or more precisely the box counting dimension) is obtained from the scaling behavior of its Hausdorff volume $H\left(\Gamma_{r}\right)$ with respect to scale $r$, the radius of balls $B_{r}$ of the covering [5,10],

$$
H\left(\Gamma_{r}\right)=\int_{B_{r}} \sqrt{1+r^{2}\left|\nabla \tilde{g}_{\alpha}\right|^{2}} \mathrm{~d}^{3} \mathbf{x} \sim r^{D_{H}} .
$$

The relative Hausdorff volume $H\left(\Gamma_{r}\right) / V_{r}$ can be estimated as

$$
\frac{H\left(\Gamma_{r}\right)}{V_{r}} \leq \sqrt{1+\frac{3}{4 \pi r} \int_{B_{r}}\left|\nabla \tilde{g}_{\alpha}\right|^{2} \mathrm{~d}^{3} \mathbf{x}}
$$


where the Cauchy-Schwartz inequality was used and $V_{r}=4 \pi r^{3} / 3$. The scalar gradient field $g_{\alpha}(\mathbf{x}, t)$ is measured in units of its root mean square (rms) value, i.e. $\tilde{g}_{\alpha}=g_{\alpha} / \sqrt{\left\langle g_{\alpha}^{2}\right\rangle_{V}}$. Here $V=L^{3}$ where $L$ is the outer scale of turbulence. Similarily, $\tilde{G}=G / \sqrt{\left\langle g_{\alpha}^{2}\right\rangle_{V}}$.

Progress is made now with a substitution of the gradient term in (3) by means of the advection-diffusion equation for the scalar gradient component,

$$
\left[\partial_{t}+u_{i} \partial_{i}-\kappa \partial_{i}^{2}\right] \tilde{g}_{\alpha}+\left(\partial_{\alpha} u_{i}\right) \tilde{g}_{i}=-\partial_{\alpha} u_{\alpha} \tilde{G} .
$$

From the resulting second order balance one gets

$$
\begin{aligned}
\left|\nabla \tilde{g}_{\alpha}\right|^{2} & =\frac{1}{2 \kappa}\left[\left(-u_{i} \partial_{i}+\kappa \partial_{i}^{2}\right) \tilde{g}_{\alpha}^{2}\right. \\
& \left.-2 \tilde{g}_{\alpha}\left(\partial_{\alpha} u_{i} \tilde{g}_{i}+\partial_{\alpha} u_{\alpha} \tilde{G}\right)\right],
\end{aligned}
$$

where summation is carried out over index $i$ only, but not over $\alpha$. The time derivative is already omitted, because we will discuss the statistically stationary case. With substitution (5), inequality (3) becomes

$$
\frac{H\left(\Gamma_{r}\right)}{V_{r}} \leq \sqrt{1+\frac{3}{4 \pi r \kappa} \int_{B_{r}}\left[\left(-u_{i} \partial_{i}+\kappa \partial_{i}^{2}\right) \frac{\tilde{g}_{\alpha}^{2}}{2}-\tilde{g}_{\alpha}\left(\partial_{\alpha} u_{i}\right) \tilde{g}_{i}-\tilde{g}_{\alpha} \partial_{\alpha} u_{\alpha} \tilde{G}\right] \mathrm{d}^{3} \mathbf{x}} .
$$

We will consider now the four integrals under the square root separately and denote them by $I_{1}, I_{2}, I_{3}$, and $I_{4}$. From (6), with incompressibility, and by applying the Gauss theorem, it follows for $I_{1}$

$$
I_{1}=\frac{3 r}{2 \kappa A_{r}} \oint_{\partial B_{r}} \tilde{g}_{\alpha}^{2}\left(\mathbf{u}-\mathbf{u}_{0}\right) \cdot \mathrm{d} \mathbf{A}
$$

$A_{r}=4 \pi r^{2}$ is the surface content of $B_{r}$ and the surface normal vector points toward the origin of $B_{r}$. It is possible to add $\mathbf{u}_{0}=\mathbf{u}\left(\mathbf{x}_{0}\right)$, the velocity at the center of $B_{r}$ for which $\left\langle\mathbf{u}_{0}\right\rangle_{\partial B_{r}}=0$. At this point, it has to be assumed that the fluctuations $\tilde{g}_{\alpha}^{2}$ are equally distributed over the sphere $\partial B_{r}$ in order to get $\oint \tilde{g}_{\alpha}^{2} \mathbf{u}_{0} \cdot \mathrm{d} \mathbf{A}=0$. The application of the Cauchy-Schwartz inequality results in

$$
I_{1} \leq \frac{3 r}{2 \kappa} \sqrt{\oint_{\partial B_{r}} \tilde{g}_{\alpha}^{4} \frac{\mathrm{d} A}{A_{r}}} \sqrt{\oint_{\partial B_{r}}\left(\left(\mathbf{u}-\mathbf{u}_{0}\right) \cdot \mathbf{n}\right)^{2} \frac{\mathrm{d} A}{A_{r}}}
$$

The first square root is a scale resolved scalar gradient flatness which will be discussed later and denoted by $F_{4}(r)=$ $\left\langle g_{\alpha}^{4}\right\rangle_{B_{r}} /\left\langle g_{\alpha}^{2}\right\rangle_{V}^{2}$. Note that the $r$-dependence comes in via coarse graining over balls of varying radius $r$. The second term stands for the second order longitudinal velocity structure function, which is $S_{\|}(r)=\epsilon r^{2} /(15 \nu)$ in the viscous range, i.e. on scales around and below the Kolmogorov dissipation scale $\eta$. We find

$$
I_{1} \leq \frac{3}{2 \kappa} \sqrt{\frac{\epsilon F_{4}(r)}{15 \nu}} r^{2}
$$

Integral $I_{2}$ in (6) can be written as

$$
I_{2}=\frac{3}{4 \pi r} \int_{B_{r}}\left[\tilde{g}_{\alpha} \nabla^{2} \tilde{g}_{\alpha}+\left|\nabla \tilde{g}_{\alpha}\right|^{2}\right] \mathrm{d}^{3} \mathbf{x}
$$

Green's formula for scalars $u(\mathbf{x})$ and $v(\mathbf{x})$,

$$
\begin{aligned}
\int_{V} u(\mathbf{x}) \nabla^{2} v(\mathbf{x}) \mathrm{d}^{3} \mathbf{x} & =\oint_{\partial V} u(\mathbf{x}) \nabla v(\mathbf{x}) \cdot \mathrm{d} \mathbf{A} \\
& -\int_{V} \nabla u(\mathbf{x}) \cdot \nabla v(\mathbf{x}) \mathrm{d}^{3} \mathbf{x},
\end{aligned}
$$

with $u(\mathbf{x})=v(\mathbf{x})=\tilde{g}_{\alpha}(\mathbf{x})$ is taken. Substitution into (10) and application of the Cauchy-Schwartz inequality result in

$$
\begin{aligned}
I_{2} & =\frac{3}{4 \pi r} \oint_{\partial B_{r}} \tilde{g}_{\alpha}\left|\nabla \tilde{g}_{\alpha}\right| \mathbf{n} \cdot \mathrm{d} \mathbf{A} \\
& \leq 3 r \sqrt{\frac{1}{A_{r}} \oint_{\partial B_{r}} \tilde{g}_{\alpha}^{2} \mathrm{~d} A} \sqrt{\frac{1}{A_{r}} \oint_{\partial B_{r}}\left|\nabla \tilde{g}_{\alpha}\right|^{2} \mathrm{~d} A} \\
& =3 r \sqrt{F_{2}(r)} \sqrt{\left\langle\left|\nabla \tilde{g}_{\alpha}\right|^{2}\right\rangle_{\partial B_{r}}}
\end{aligned}
$$


with $F_{2}(r)=\left\langle g_{\alpha}^{2}\right\rangle_{\partial B_{r}} /\left\langle g_{\alpha}^{2}\right\rangle_{V}$ and $\nabla \tilde{g}_{\alpha}=\left|\nabla \tilde{g}_{\alpha}\right| \mathbf{n}$. We assume that the volume average as well as the surface average give the same results over scales $r$ due to homogeneity of turbulence.

The third integral, $I_{3}$, can be estimated by the Cauchy-Schwartz inequality to

$$
\begin{aligned}
I_{3} & =-\frac{3}{4 \pi r \kappa} \int_{B_{r}} \tilde{g}_{\alpha}\left(\partial_{\alpha} u_{i}\right) \tilde{g}_{i} \mathrm{~d}^{3} \mathbf{x} \\
& \leq \frac{r^{2}}{\kappa} \sum_{i=1}^{3} \sqrt{\left\langle\tilde{g}_{\alpha}^{2} \tilde{g}_{i}^{2}\right\rangle_{B_{r}}\left\langle\left(\partial_{\alpha} u_{i}\right)^{2}\right\rangle_{B_{r}}} .
\end{aligned}
$$

It is reasonable to assume that the fluctuations along the mean scalar gradient are the largest so that the mixed terms can be estimated by $\left\langle\tilde{g}_{\alpha}^{2} \tilde{g}_{i}^{2}\right\rangle_{B_{r}} \leq\left\langle\tilde{g}_{\alpha}^{4}\right\rangle_{B_{r}}=F_{4}(r)$ for $i \neq \alpha$. The velocity gradient can be treated by the energy dissipation averaged over balls $B_{r}$. Here we estimated $\sum_{i=1}^{3}\left\langle\left(\partial_{\alpha} u_{i}\right)^{2}\right\rangle_{B_{r}} \leq\langle\epsilon\rangle_{B_{r}} / \nu$. The notation $\epsilon_{r}=\langle\epsilon\rangle_{B_{r}}$ is used for the following and consequently

$$
I_{3} \leq \frac{r^{2}}{\kappa} \sqrt{\frac{F_{4}(r) \epsilon_{r}}{\nu}}
$$

Similarily one can proceed for the integral $I_{4}$

$$
I_{4}=-\frac{3}{4 \pi r \kappa} \int_{B_{r}} \tilde{g}_{\alpha}\left(\partial_{\alpha} u_{\alpha}\right) \tilde{G} \mathrm{~d}^{3} \mathbf{x} \leq \frac{r^{2} \tilde{G}}{\kappa} \sqrt{\frac{F_{2}(r) \epsilon_{r}}{\nu}} .
$$

In summary we get

$$
\frac{H\left(\Gamma_{r}\right)}{V_{r}} \leq \sqrt{1+\frac{3 r^{2}}{2 \kappa} \sqrt{\frac{\epsilon F_{4}(r)}{15 \nu}}+3 r \sqrt{F_{2}(r)\left\langle\left|\nabla \tilde{g}_{\alpha}\right|^{2}\right\rangle_{\partial B_{r}}}+\frac{r^{2}}{\kappa} \sqrt{\frac{F_{4}(r) \epsilon_{r}}{\nu}}+\frac{r^{2} \tilde{G}}{\kappa} \sqrt{\frac{F_{2}(r) \epsilon_{r}}{\nu}}} .
$$

Unfortunately left with a couple of unknown terms in this expression. Further progress can be made only by the use of dimensional arguments. One can expect that the energy dissipation field fluctuates most strongly around scale $\eta$ and large velocity gradients will be smoothed by the finite viscosity on scales below. Therefore it is reasonable to assume

$$
\frac{\epsilon_{r}}{\epsilon} \leq \frac{\epsilon_{\eta}}{\epsilon} \simeq\left(\frac{\eta}{L}\right)^{\gamma-1}=\left(\frac{20}{3} R_{\lambda}^{-2}\right)^{\frac{3}{4}(\gamma-1)}
$$

where $(\eta / L)=(20 / 3)^{3 / 4} R_{\lambda}^{-3 / 2}[11] . \gamma$ is a (universal) scaling exponent varying between $\gamma_{1}$ and $\gamma_{2}$. Clearly, the minimum exponent, $\gamma_{1}$, will be the dominant one.

The following term in $I_{2}$, can be simplified to

$$
\left\langle\left|\nabla \tilde{g}_{\alpha}\right|^{2}\right\rangle_{\partial B_{r}} \leq \frac{1}{\eta_{B}^{2}}\left\langle\left(\tilde{g}_{\alpha}\right)^{2}\right\rangle_{\partial B_{r}}=\frac{F_{2}(r)}{\eta_{B}^{2}},
$$

and $F_{2}(r)$ follows in lines with (17) to

$$
F_{2}(r)=\frac{\left\langle g_{\alpha}^{2}\right\rangle_{\partial B_{r}}}{\left\langle g_{\alpha}^{2}\right\rangle_{V}} \simeq \frac{\epsilon_{\theta, r}}{\epsilon_{\theta}} \leq \frac{\epsilon_{\theta, \eta_{B}}}{\epsilon_{\theta}} \simeq\left(\frac{\eta_{B}}{L}\right)^{\delta-1},
$$

where $\epsilon_{\theta, r}=\left\langle\epsilon_{\theta}\right\rangle_{\partial B_{r}}$ is the coarse grained scalar dissipation rate. With $\eta_{B} / \eta=S c^{-1 / 2}$ one gets

$$
F_{2}(r) \leq\left[\left(\frac{20}{3}\right)^{\frac{3}{4}} S c^{-\frac{1}{2}} R_{\lambda}^{-\frac{3}{2}}\right]^{\delta-1} .
$$

Again the scaling exponent $\delta \in\left[\delta_{1}, \delta_{2}\right] . F_{4}(r)$ can be estimated as follows

$$
F_{4}(r)=\frac{\left\langle g_{\alpha}^{4}\right\rangle_{B_{r}}}{\left\langle g_{\alpha}^{2}\right\rangle_{V}^{2}}=\frac{\left\langle g_{\alpha}^{4}\right\rangle_{B_{r}}}{\left\langle g_{\alpha}^{4}\right\rangle_{V}} F_{4}(L) \leq\left(\frac{L}{\eta_{B}}\right)^{4} F_{4}(L)
$$


where $F_{4}(L)=\left\langle g_{\alpha}^{4}\right\rangle_{V} /\left\langle g_{\alpha}^{2}\right\rangle_{V}^{2},\left\langle g_{\alpha}^{4}\right\rangle_{B_{r}} \leq G^{4} / \eta_{B}^{4}$, and $\left\langle g_{\alpha}^{4}\right\rangle_{V} \simeq G^{4} / L^{4}$. G is the magnitude of the mean scalar gradient. It is also assumed that scalar gradient moments are at maximum around the Batchelor scale. Thus follows

$$
F_{4}(r) \leq \frac{27}{8000} S c^{2} R_{\lambda}^{6} F_{4}(L) .
$$

The term $I_{3}$ will be much larger than $I_{4}$ if for the mean scalar gradient holds, $\tilde{G} \ll \sqrt{F_{4}(r) / F_{2}(r)}$, as it will be the case for the comparison with numerical experiments. Finally, $I_{1}$ will always be subdominant compared to $I_{3}$ due to $\epsilon_{r} \gg \epsilon$.

All the estimates are inserted now into (16) and we assume a scaling relation for the relative Hausdorff volume, $H\left(\Gamma_{r}\right) / V_{r} \sim r^{D_{H}-3}$. Additionally, all scales are expressed in units of Kolmogorov dissipation length, $\tilde{r}=r / \eta$. The Hausdorff dimension of the graph over two-dimensional balls is given by the additive law, $D_{H}^{\prime}=D_{H}-1$, [12] when assuming almost isotropic graphs,

$$
\begin{aligned}
D_{H}^{\prime} & =2+\frac{\mathrm{d} \ln \left(H\left(\Gamma_{\tilde{r}}\right) / V_{\tilde{r}}\right)}{\mathrm{d} \ln \tilde{r}} \\
& \leq 2+\frac{\mathrm{d}}{\mathrm{d} \ln \tilde{r}} \ln \sqrt{1+3\left[\left(\frac{20}{3}\right)^{\frac{3}{4}} R_{\lambda}^{-\frac{3}{2}}\right]^{\delta_{1}-1} S c^{\frac{2-\delta_{1}}{2}} \tilde{r}+\sqrt{\frac{27 F_{4}(L)}{8000}}\left(\frac{20}{3}\right)^{\frac{3}{8}\left(\gamma_{1}-1\right)} S c^{2} R_{\lambda}^{\frac{15-3 \gamma_{1}}{4}} \tilde{r}^{2} .}
\end{aligned}
$$

The expression probes the local slope of the scaling relation. The upper bound of $D_{H}^{\prime}$ will be determined by the leading term under the square root at every scale $\tilde{r}$ and we expect the result $2 \leq D_{H}^{\prime} \leq 3$.

The missing scalar derivative flatness factor $F_{4}(L)$ can be evaluated from [1] and present simulations. The latter are conducted in a homogeneously sheared flow in which the scalar field of constant gradient was allowed to evolve according to the advection-diffusion equation [4,13]. The mean scalar gradient is kept the same in all runs resulting in dimensionless $\tilde{G}=0.37$ at $S c=1$ and 0.05 at $S c=64$ so that $\tilde{G} \ll \sqrt{F_{4}(r) / F_{2}(r)}$ is justified. As the inset of Fig. 2 shows, the flatness factors for both simulations are $\sim 10$ for $S c>1$ and will be taken constant for the following.

The two minimum scaling exponents, $\gamma_{1}$ and $\delta_{1}$, can be taken from experiments where the whole multifractal spectrum for $\epsilon_{\theta}(\mathbf{x}, t)$ and $\epsilon(\mathbf{x}, t)$ was measured, respectively. Meneveau and Sreenivasan [14] found for experimental data at different Reynolds numbers that $\gamma_{1}$ is about $1 / 4$. Prasad et al. found a value of $\delta_{1} \approx 2 / 5$ in a high-Schmidt number scalar mixing experiment at $S c \approx 2000$ [15].

Figure 2 plots (23) for three different values of $S c$. For scales accessible to the simulations, i.e, larger than $\eta_{B}$ the upper bound is found to be always 3 . While for the scalar case the crossover of $D_{H}^{\prime}$ from 2 to 3 takes place around $\eta_{B}[8]$, it is present here for by far smaller scales. It is just the fairly rough estimate for the additional scalar gradient stretching term (last term on the l.h.s. of (4)) that causes the crossover scale, $\tilde{r}_{c}$, to be smaller than $\eta_{B}$. When inserting the numbers, one gets $\tilde{r}_{c} \sim S c^{-6 / 5} R_{\lambda}^{-213 / 80}$, at which the second term of (23) starts to dominate. To illustrate this effect, we kept $I_{1}$ only and plot the corresponding $D_{H}^{\prime}$ as dashed lines in Fig. 2. The bounds are shifted then by an order of magnitude, but still rough due to estimate (22).

For comparison, we conducted the relative Hausdorff volume of the scalar gradient field from numerical simulation data. Two-dimensional "balls" are then squares of a dyadic grid of sidelength $2^{-j} \times 2 \pi$. The corresponding local slope of the scaling dimension is added in Fig. 2 and a plateau with a non-integer dimension of $D_{H}^{\prime} \approx 2.3$ can be observed for intermediate scales. This feature cannot be reproduced by the upper bounds because the $\tilde{r}$-dependence of all four $I$ terms has integer powers only. Even for passive scalars, non-integer $D_{H}$ were obtained only for inertial range scaling of the velocity structure function of $\sim r^{2 / 3}$ (cf. [8]).

To summarize, we have discussed the applicability of the geometric measure theory to scalar gradient fields for high-Sc mixing. The upper bounds on $D_{H}$ are consistent with simulation results but rough. Additionally, they are insensitive to the particular choice of the threshold value of the level set and thus cannot capture slight differences between positive and negative level sets of same magnitude which were discussed in [4]. Interestingly, a dependence of the bounds on the Taylor-Reynolds number results. Batchelor's original model for the visocous-convective range was thought to be insensitive to the physics in the inertial range, i.e., on scales larger $\eta$ [9]. A detailed investigation of that problem will be a part of future work and further improvement and extension of the bounds might be possible therefore.

The numerical computations were carried out on the IBM Blue Horizon at the San Diego Supercomputer Center within the NPACI program of the US National Science Foundation which we wish to acknowledge. Comments by C. R. Doering, B. Eckhardt, and K. R. Sreenivasan are acknowledged. 
[1] P. K. Yeung, S. Xu, and K. R. Sreenivasan, Phys. Fluids 14, 4178 (2002).

[2] G. Brethouwer, J. C. R. Hunt, and F. T. M. Nieuwstadt, J. Fluid Mech. 474, 193 (2003).

[3] Z. Warhaft, Annu. Rev. Fluid Mech. 32, 203 (2000).

[4] J. Schumacher and K. R. Sreenivasan, Phys. Rev. Lett. 91, 174501 (2003).

[5] F. Morgan, Geometric Measure Theory, a Beginners Guide, (Academic Press, Boston) 1988.

[6] P. Constantin, I. Procaccia, and K. R. Sreenivasan, Phys. Rev. Lett. 67, 1739 (1991).

[7] P. Constantin and I. Procaccia, Phys. Rev. E 47, 3307 (1993).

[8] S. Grossmann and D. Lohse, Europhys. Lett. 27, 347 (1994); B. Eckhardt and J. Schumacher, Phys. Rev. E 60, 4185 (1999).

[9] G. K. Batchelor, J. Fluid Mech. 5, 113 (1959).

[10] K. J. Falconer, The Geometry of Fractal Sets, Cambridge University Press, Cambridge 1985.

[11] S. B. Pope, Turbulent flows, Cambridge University Press, Cambridge 2000.

[12] P. Mattila, Ann. Acad. Sci. Fenn. Ser. A. Math. 1, 227 (1975).

[13] J. Schumacher and B. Eckhardt, Europhys. Lett. 52, 627 (2000); J. Schumacher, J. Fluid Mech. 441,109 (2001).

[14] C. Meneveau and K. R. Sreenivasan, Phys. Rev. A 38, 6287 (1989).

[15] R. R. Prasad, C. Meneveau and K. R. Sreenivasan, Phys. Rev. Lett. 61, 74 (1988).

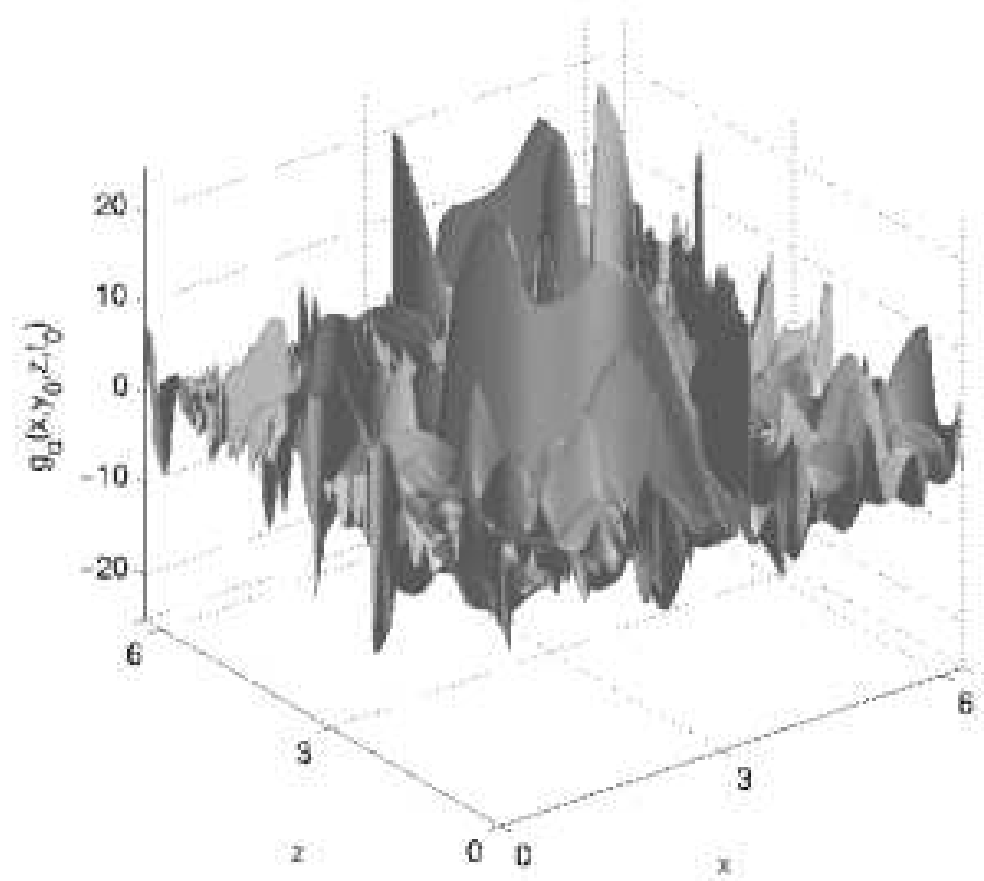

FIG. 1. The graph of the scalar gradient field $g_{\alpha}$ which is shown here over the $x-z$ plane at fixed $y_{0}$ and $t_{0}$ for numerical data at $S c=16$. Grid resolution was $N=512$. 


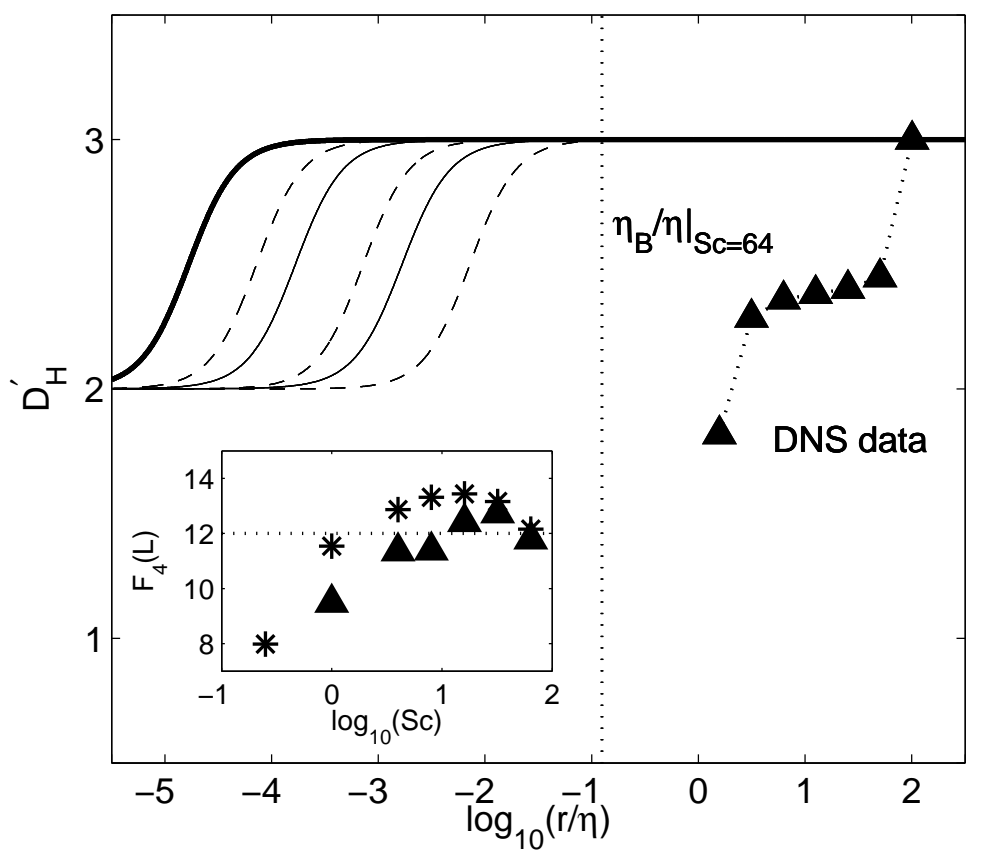

FIG. 2. Scale dependent upper bound of the Hausdorff dimension of scalar gradient level sets for different Schmidt numbers. Value of $R_{\lambda}=87$ is taken from [4]. Solid lines are for $S c=64$ (thick line), 6.4, and 0.64 from left to right. Dashed lines are for bounds at the same $S c$ if integral $I_{1}$ would dominate in (23) as in the scalar case [8]. The Batchelor scale for $S c=64$ is marked as a dotted vertical line. The panel contains also the DNS findings for the scale resolved $D_{H}^{\prime}$ for $S c=64$ with a plateau at a value of about 2.3 . Inset: Flatness factor $F_{4}(L)$ of the scalar derivative along the mean scalar gradient, $g_{\alpha}$, as a function of the Schmidt number. The dotted line stands for the mean of all data points and is drawn at 12 . Present data are indicated by triangles and data from [1] by asterisks. 